Article

\title{
Contributorship, Not Authorship: Use CRediT to Indicate Who Did What
}

\author{
Alex O. Holcombe ${ }^{(D)}$ \\ The University of Sydney, Sydney 2006, Australia; alex.holcombe@sydney.edu.au
}

Received: 21 May 2019; Accepted: 27 June 2019; Published: 2 July 2019

\begin{abstract}
Participation in the writing or revising of a manuscript is, according to many journal guidelines, necessary to be listed as an author of the resulting article. This is the traditional concept of authorship. But there are good reasons to shift to a contributorship model, under which it is not necessary to contribute to the writing or revision of a manuscript, and all those who make substantial contributions to a project are credited. Many journals and publishers have already taken steps in this direction, and further adoption will have several benefits. This article makes the case for continuing to move down that path. Use of a contributorship model should improve the ability of universities and funders to identify effective individual researchers and improving their ability to identify the right mix of researchers needed to advance modern science. Other benefits should include facilitating the formation of productive collaborations and the creation of important scientific tools and software. The CRediT (Contributor Roles Taxonomy) taxonomy is a machine-readable standard already incorporated into some journal management systems and it allows incremental transition toward contributorship.
\end{abstract}

Keywords: authorship; incentives; funding; Meta-science

\section{Introduction}

Classically, an author is someone who writes something. The first definition of "author" listed in many dictionaries states that writing is a necessary condition for authorship (e.g., according to the Cambridge English Dictionary, an author is "the writer of a book, play, article, etc."). When in the early modern period scholarly communication was formalized as articles in scientific journals, the practice of giving scientific credit to authors of scientific articles and letters served science well. When it is individuals working alone that make scientific discoveries and that write up the formal accounts, credit to the writers of those accounts is where credit is due. In the Philosophical Transactions of the Royal Society in 1752, for example, almost every article was credited to just one author.

Science today is highly collaborative, and results usually reflect the work of multiple people. Many of those who do this work are evaluated on their contributions to science when they apply for a job, a promotion, or a grant. One might expect this evaluation to be based simply on one's contributions to science. In many fields, evaluation is formally based on the list of publications that include the researcher as an author. But there can be a disconnect between those who are authors and those who make contributions. While author typically means "writer", funders and promotion committee members are interested in various types of substantive contributions-who actually provided the words in the paper is, often, not the paramount concern.

Many journals and societies specifically enshrine manuscript drafting or revising as a requirement for co-authorship. This is a problem for funders and others interested in both writing-related contributions and also contributions that are not writing related.

One of the most influential authorship policies is that formulated by the International Committee of Medical Journal Editors (ICMJE), e.g., [1]. "Drafting the work or revising it critically for important 
intellectual content" is a requirement to qualify as a potential journal article author, according to the ICMJE. I will refer to the ICMJE's and others' criteria that stipulate that contributing to writing (in any way) is necessary for authorship as "writing-mandatory". The guidelines of the American Psychological Association, which publishes several dozen journals, are also writing-mandatory—all authors must be involved in "manuscript drafting" [2].

The requirement to participate in drafting or other aspects of writing puts many researchers in a difficult position. Often a manuscript reports the outcome of a study that involved crucial contributions from many individuals. While only one or a few might, for various reasons, make significant contributions to the writing of the manuscript, these manuscript writers know that their collaborators' career success may depend on them becoming co-authors. In other words, researchers need to be recognized for substantial contributions, even when these contributions were not to the writing-what has been termed their contributorship rather than authorship [3].

This need for formal recognition is in part why, despite guidelines that proscribe the practice, researchers frequently include as co-authors people who made a substantial contribution in any of various areas, even if they did not contribute to the writing. Practically, then, the horse may have already bolted on the issue of whether to drop the writing mandate from authorship criteria; that is, if authorship guidelines are to be in line with actual practice, then a contribution to writing should today be neither required nor mandatory.

More broadly, the concept of authorship itself may no longer be appropriate. With standard practice today, the ambiguity regarding the contribution(s) of a co-author to a paper extends well beyond whether there was a contribution to the writing. Surveys of researchers show strong disagreement both with the most widely used authorship guidelines and also high levels of inconsistency between researchers regarding the categories of contributions that merit authorship. A large multi-national study [4] found that for almost every category of researcher contribution to a project, opinions are highly variable, such that as many people think that a particular contribution category should rarely ever merit authorship as those that think it should almost always merit it. For example, while some researchers feel that securing funding or establishing the team of researchers "almost always" merits authorship, approximately equal numbers feel that it "almost never" does. Given that many of these researchers likely act on these beliefs, being an author is not currently a clear signal of having satisfied any particular criteria.

To resolve the ambiguity in the meaning of author lists, one would have to ask the authors what the contributions were of each author and what, if any, were the significant contributions of others. This is the heart of the contributorship concept. Under a contributorship model, authors are required to state this information. And in a world where a number of researchers often contribute one or more of a variety of things to a research project, it seems illogical for researchers not to say something about who did what in a way that is visible and systematically enables credit for those contributions.

The transparency and removal of ambiguity stemming from a declaration of contributions will have multiple benefits. Universities, laboratories, and institutions make hiring and funding decisions based on what they can determine about a person's past scientific contributions. These hiring organizations can have very different interests in specific kinds of contributions. When hiring a staff scientist in a large laboratory, or a statistician in a research group, one is looking for different types of contributions than if one is hiring a faculty member expected to run a laboratory. Thus, to best serve the various stakeholders involved in science, what is needed is to know what the significant contributions were to a project and who made them.

Under a contributorship model, all those who make substantial contributions are indicated and the nature of each named person's contributions listed [4,5]. If captured in a structured format, such contributions become visible at scale, providing valuable information to support researchers, universities, funders, and societies. The named contributors need not have contributed to the writing of the paper. Note that a contributorship model does not hinder those who, for whatever reason or purpose, do not wish to take into account researchers who do not contribute to the writing. Such people 
could generate lists of papers that a particular researcher contributed to the writing of while excluding other papers the researcher made other types of contributions to.

The purpose of this paper is both to present the case for adopting a contributorship model, and to describe practical paths to adoption. Many journals and publishers are already partway down one path - a path endorsed in a recent Proceedings of the National Academy of Science article by McNutt et al. [6]. Because the specifics of the McNutt et al. proposal has already met with some success and is an initiative that can be rallied around to drive change, I will focus on it.

\section{The Move to a Contributorship Model}

In their paper entitled "Transparency in authors' contributions and responsibilities to promote integrity in scientific publication", McNutt et al. [6] called for journals to move to a contributorship model, although they did not put it that way. Along with the National Academy of Sciences president Marcia McNutt, the article's authors include those who were or had been the chief editor of eLife, the chief editor of the New England Journal of Medicine, an editor of Science, and an editor of Cell.

The McNutt et al. paper [6] has two primary recommendations. First, that journals use the CRediT (Contributor Roles Taxonomy, http://www.casrai.org/credit.html) taxonomy to record what individuals contribute to scientific papers. Second, that publishers and societies change their criteria for authorship. The changes they propose would remove the writing mandate from common authorship guidelines, which in combination with CRediT amounts to perhaps a full shift to a contributorship model. However, McNutt et al. do not use the word "contributorship" in their paper. They do not address contributorship directly by any name or describe the case for it from basic principles of credit. Their paper is very brief and simply couches their suggestions as potential improvements to authorship guidelines and practices. As a result of this presentation, readers are unlikely to realize the full significance of the recommendations, which is one gap this article aims to fill. In particular, McNutt et al. concentrated on how their recommendations could combat bad practices that are already sanctioned by existing guidelines, such as "honorary authorship". The present article concentrates on the positive benefits expected rather than the elimination of abuses.

\section{Authorship Criteria: ICMJE Versus Contributor Roles}

An advantage of presenting authorship criteria as a simple change to ICMJE criteria, as McNutt et al. did [6], is that it makes the guidelines easy for journals and societies to support, but I believe this obfuscated the extent of the implications of the proposed changes. McNutt et al. discussed their guidelines only briefly, as "adapted from" the International Committee of Medical Journal Editors (ICMJE) guidelines and "generalized to encourage broader adoption". From that description, many readers will fail to realize that the guidelines include a very significant change: They are not writing-mandatory; that is, contributing to the writing of a paper is no longer necessary to qualify for authorship.

Box 1 shows the McNutt et al. [6] authorship guidelines.

Both the ICMJE [1] and McNutt et al. [6] criteria have two parts, as do the authorship criteria of many other organizations. One part (in most cases, the second part) is not concerned with actual contributions. Instead it is about approval of the final manuscript and agreement to be accountable for the work. This part is very similar in ICMJE and McNutt et al. In this part, McNutt et al. make only a small change, to release authors from all having to approve small changes to the final version of a manuscript, which often arise at the "proofs" stage. 
Box 1. The proposed McNutt et al. criteria [6], and the traditional, widely adopted ICMJE [1] criteria. Boldface has been added to indicate an important difference, which is discussed in the text.

McNutt et al. criteria [6]

1. Each author is expected to have made substantial contributions to the conception or design of the work; OR the acquisition, analysis, OR interpretation of data; OR the creation of new software used in the work; OR have drafted the work or substantively revised it; AND

2. to have approved the submitted version (and any substantially modified version that involves the author's contribution to the study); AND

3. to have agreed both to be personally accountable for the author's own contributions and to ensure that questions related to the accuracy or integrity of any part of the work, even ones in which the author was not personally involved, are appropriately investigated, resolved, and the resolution documented in the literature.

ICMJE criteria (2018 update)

1. Substantial contributions to the conception or design of the work; or the acquisition, analysis, or interpretation of data for the work; AND

2. Drafting the work or revising it critically for important intellectual content; AND

3. Final approval of the version to be published; AND

4. Agreement to be accountable for all aspects of the work in ensuring that questions related to the accuracy or integrity of any part of the work are appropriately investigated and resolved.

The other part of most authorship guidelines typically appears first and is about the contributions of the authors. This part contains the clause that makes the ICMJE guidelines writing-mandatory. It specifies that fulfilling its writing-related criterion is necessary to become a co-author. Specifically, authors must participate in "Drafting the work or revising it critically for important intellectual content." Finally, an author must also contribute "substantively" to another aspect of the work, which can be any of the following: "the conception or design of the work; or the acquisition, analysis, or interpretation of data".

In the McNutt et al. guidelines (Box 1), satisfying the writing-related criterion is not necessary for co-authorship. This is a result of McNutt et al. using the word "or" rather than "and" in association with the writing-related criterion. Elimination of the necessity for a writing-related contribution brings a journal closer to a contributorship model, which names all those who made substantial contributions. A researcher who made a substantial contribution such as analysing the data, creating a critical piece of custom equipment, or writing software that conducts the experiment can become a co-author on the paper without contributing to the writing.

Today, there are many prominent journals, as well as entire fields, using guidelines that are not writing-mandatory. In some cases, the journals adopted the McNutt et al. recommendations, and in other cases the practice is more long-standing. For example, the guidelines of the Nature Publishing Group [7], the Ecological Society of America (https://www.esa.org/esa/about/governance/esa-code-ofethics/), and the American Chemical Society (http://pubs.acs.org/paragonplus/submission/jacsat/jacsat_ authguide.pdf) do not require writing-related contributions. The American Chemical Society has long had a guideline that an author need only make "significant scientific contributions to the work". And it seems that chemistry journals and Nature journals have not been reported to receive complaints that researchers are getting their names on papers without contributing to the writing. Although in this way journals in chemistry and ecology are aligned with the contributorship model, they traditionally have not required researchers to specify what contribution(s) they made to a paper. More recently, however, many journals have started requiring an explicit description of what an author's contribution is [8]. The Nature Publishing Group journals, for example, made these descriptions mandatory, and after a ten-year trial saw rapid voluntary uptake of the option [9]. However, as will be described below, to realize the full benefits of contributorship, standardization is required. 


\section{Benefits of a Contributorship Model with a Standardized Taxonomy of Contributions}

As mentioned above, many journals today require an author contributions section indicating which author did what. To realize most of the potential benefits described below, this would have to occur at a larger scale, and moreover, standardization is arguably necessary for substantive assessment of anything more than small numbers of publications. This section elaborates on some anticipated benefits if a standardized contributorship system were adopted by large numbers of journals. The subsequent section then details the standardized system, CRediT, that has already gained traction.

Some benefits of adopting a standardized contributorship system:

1. A reduction in honorary authorship and the ambiguity of researcher contributions.

2. Those interested in specific kinds of contributions can assess researchers on that specific basis.

3. Cross-disciplinary and cross-subfield collaborations will be facilitated.

4. The development of scientific software will be facilitated.

5. The contributions of statisticians and others in "specialist roles" will be more appropriately recognized (and eventually, rewarded).

6. Meta-science will be greatly facilitated.

(1) A reduction in honorary authorship and in the ambiguity of researcher contributions. The ambiguity that results from conventional authorship practices was mentioned in the Introduction. That ambiguity is greatly reduced by contributorship.

Many previous authors have suggested that requiring contributions to be spelled out reduces "honorary" authorship, in which researchers (typically senior ones) are listed as co-authors despite not satisfying the extant criteria [10-13]. This may be true, and superficially this would be good because honorary authorship is a dishonest practice. However, it is important to consider that there may be reasons to acknowledge the contributions of some who currently receive only dishonest honorary authorship.

Leaders of large groups may spend nearly all of their time securing funding or in administrative tasks. Perhaps it is appropriate for such people to receive some credit for this, because at least in the current system, it can be an important role. Because the CRediT contributorship taxonomy includes separate "Funding acquisition", "Project administration", "Supervision", and "Resources" contribution types, adoption of CRediT (or a system like it) allows researchers to be listed on papers without having to claim that they contributed to the writing of the paper, which is anticipated to stem false claims of contributions. Those who wish to disregard such contributions may do so, for example by generating lists of authors who contributed to more than those categories.

(2) Those interested in specific kinds of contributions can assess researchers on that specific basis. The Introduction of this paper pointed out universities' interest in hiring the appropriate person, or combination of persons, for the increasingly specialized enterprise of science. This can come down to a certain mix of contribution types. Today services such as PubMed, Google Scholar, and ORCiD author pages provide only a simple list of papers that authors are an author on. These services will be enhanced or new ones, e.g., CurateScience.org, will become more prominent for organizing researcher contributions based on their type. Users will be able to view the papers a researcher or group of researchers contributed to, organized by type of contribution or number of contributions. The technical obstacles to this are few because such databases and services already make use of the JATS XML metadata where the CRediT information is stored.

(3) Cross-disciplinary and cross-subfield collaborations will be facilitated. Consider a project that is based in one discipline, but has substantial inter-disciplinary contributions. For example, a neuroscience project might benefit from a fairly new mathematical technique to analyse multivariate time-series data. Let us say that the contributors to the project know of only one person, a mathematician, who understands the technique in any depth. Assume that the mathematical technique is already described in a paper, and citing that article would suffice to indicate how it were used for the neuroscience study. Thus, the mathematician does not need to contribute to the writing of the article. 
The mathematician may also not have time to become familiar with how the psychologists describe the project, and even if she did, she might still not be able to contribute to the writing of the article, or to anything other than the data analysis aspect of the project. In an area with writing-mandatory authorship guidelines, the mathematician would not qualify for authorship, giving her little to no career incentive to help with the project.

The relatively low incentive for researchers to contribute techniques to a project in another discipline likely slows the dissemination of techniques from mathematics to science and from one field of science to another. Throughout history science has benefited from developments in mathematics, but knowledge transfer across disciplines often takes decades [14] — this is one reason that funders have called for more interdisciplinary collaboration $[15,16]$. The CRediT contribution taxonomy includes a "Formal analysis" contribution category, which is described as "Application of statistical, mathematical, computational, or other formal techniques to analyse or synthesize study data", which would fit the mathematician's contribution in our example. This relationship with the mathematician may then, of course, extend beyond the immediate project, with the mathematician, now somewhat familiar with the project, extending the technique for follow-up projects, or recognizing other areas where additional mathematical techniques could help.

This sort of situation is not confined to cross-disciplinary collaborations, but also arises with researchers in different sub-fields of areas like molecular biology. A researcher may recognize that a technique from his discipline would benefit another researcher that he has met, and that it would be straightforward for the researcher to do the relevant work. For example, the researcher might be highly skilled in separating certain compounds, although quite unfamiliar with other aspects of the project or the subfield in which it lies. Without the ability to separate those compounds, the project would not succeed. However, the researcher may not meet conventional authorship criteria because, like the mathematician in the previous example, they may not have the expertise in the subfield necessary to make writing-related contributions. Or, simply out of convenience or by mistake, the leaders of the project may not involve this more peripheral researcher in the writing of the manuscript. But in a contributorship system like CRediT, this contribution could be recognized by the "Investigation" contribution type, described as "Conducting a research and investigation process, specifically performing the experiments, or data/evidence collection."

(4) The development of scientific software will be facilitated. Much of science today relies on computer code, which is sometimes written specifically for a scientific project. Not infrequently, this code extends to hundreds or thousands of lines of code. Unfortunately, writing such code does not qualify a researcher for authorship under writing-mandatory authorship criteria.

Writing the code for a project often involves a skill set distinct from that needed for other aspects of a project. Moreover, writing the code may not require much knowledge of other aspects of a project. Strong scientific programming skills can also enable one to make valuable contributions to an array of fields, too wide an array for an individual to become knowledgeable enough to understand much of the content of each. These factors suggest that those who write code are both likely to specialize in programming, but also be particularly prone to not being recognized as authors.

If programmers are under-recognized due to the prevailing authorship criteria, the proportion of funding going to programmers is likely to be lower than is optimal for scientific progress. As a result, many scientists are forced to spend time learning coding skills rather than capitalizing on their core expertise. In my own field, programming is often the rate-limiting step for conducting experiments, as only a small minority of graduate students begin their degree with the programming skills needed to code experiments. If those who program code for a study were formally recognized as contributors, this might result in a more appropriate level of funding of such people. The CRediT taxonomy provides for formal recognition with a "software" contribution type, described as "Programming, software development; designing computer programs; implementation of the computer code and supporting algorithms; testing of existing code components". 
(5) More appropriately reward the contributions of statisticians and other "specialist roles". In most areas of science, the statistics calculated are critical to the inferences made, and like programming, understanding and doing statistics often involves training independent of the training in a field's subject matter. Sometimes the actual writing of the paper does not require input from the statistician. In such cases, if the primary authors of a manuscript do not make an effort to include the statistician in the writing of the paper, they will not meet writing-mandatory authorship criteria. And unfortunately, some statisticians believe that "complaining about this (not being given the opportunity to be an author) can make you a pariah or get you a reputation as being 'difficult' (for the outrageous demand of wanting credit for your work...)" [17]. The adoption of guidelines that are not writing-mandatory should result in more statisticians being formally recognized in papers. Adoption of a standard taxonomy such as CRediT to indicate researchers' contributions is likely to result in a further increase.

Without a contributorship taxonomy, even with authorship guidelines that are not writing-mandatory, the primary authors of a paper may, with understandable attachment to old norms, think "does the statistician really deserve authorship for doing the statistics?". An advantage of spelling out contributions is that authors can include the statistician while clearly indicating that their only contribution was to the statistics. The further advantage of a taxonomy is that authors can be more secure in the knowledge that those who, in whatever context recognize analysis contributions as not important, can still assess large numbers of papers and researchers for non-analysis contributions. Feelings that a statistician might unfairly get their names on more papers than other researcher types should subside.

(6) Meta-science will be greatly facilitated. As scientific teams have become larger and more complex, the question of what mix of researcher types would most efficiently advance science has become harder to answer. Large-scale empirical analysis of the literature presently does not provide much information because the contributions of researchers are not spelled out, many contributors are not named in the author list, and those contributions that are spelled out cannot be easily tabulated by machines. Grant agencies, universities, and national laboratories will be able to make better decisions by analysing the sorts of teams that deliver the best research. Meta-scientists will be able to explore how different types of researchers add to the mix as well as better understand the gender and career-level distribution of contributions to both high-impact and low-impact work. In addition, the need for large collaborative, multi-disciplinary projects to address certain challenges and research questions in science suggests that it is paramount to recognize the contributors to large initiatives in a fairly granular way. The UK Academy of Medical Sciences has been a vocal supporter of the CRediT taxonomy as a way to help incentivise researchers to join large teams with the know-how to do rigorous, high-quality work [18].

\section{Standardized Contributorship}

In a standardized contributorship system, not only are all those who contributed substantially to a project listed on the article, with an indication of what each did, but they are also listed in a form that lends itself to large-scale analysis. Knowing who did what across large numbers of scientific papers will allow funders to better reward or fund different types of scientific contributions with the right mix. The contribution descriptions required by, for example, the Nature Publishing Group journals allow this in principle, but because the journals do not follow a standard shared by other publishers, the data cannot easily be analysed by machines across journals with different publishers. However, in part due to the endorsement by McNutt et al. [6], the CRediT taxonomy has been adopted by several scholarly publishing outlets as a practical and implementable standard that has been tested by the community, has a specified meta-data standard (JATS for xml. 1.2), and can evolve over time.

CRediT was created by a group of journal editors and scholars hosted by the Wellcome Trust and Harvard University who first met in 2012 (https://forum.casrai.org/t/origins-of-the-contributorrole-taxonomy-credit/1855) [19,20]. It includes a standard for including contributor information in the JATS-XML metadata used for scientific articles. CRediT is now in use by more than two dozen publishers, 
including Cell Press, PLoS, and Springer. It is implemented by the journal management systems or associated platforms of Aries' Editorial Manager, River Valley's ReView, the Coko Foundation, Gates Open Research, and Wellcome Open Research. Each organization allows the authors of a manuscript to enter the contribution type of each author by ticking options on a checklist (see Figure 1), as well as optionally specifying a contribution in more detail with free text.

\begin{tabular}{|c|c|c|}
\hline \multirow[b]{2}{*}{ 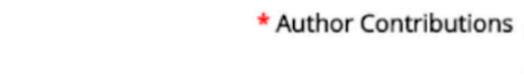 } & \multicolumn{2}{|l|}{ (At least 1 is required.) } \\
\hline & $\square$ Conceptualization & Resources \\
\hline We follow the recommendations of the ICMJE & $\square$ Data curation & $\square$ Software \\
\hline Please indicate the author's contributions: & $\square$ Formal analysis & $\square$ Supervision \\
\hline & Funding acquisition & $\square$ Validation \\
\hline & $\square$ Investigation & $\square$ Visualization \\
\hline & Methodology & $\square$ Writing - original draft \\
\hline & $\square$ Project administration & Writing - review and editing \\
\hline & \multicolumn{2}{|l|}{ Click here to add more detailed descriptions (optional) } \\
\hline & $\begin{array}{l}\text { Developed the binding assay; developed off-rate } \\
\text { assays and performed measurements }\end{array}$ & \\
\hline
\end{tabular}

Figure 1. The interface of the journal eLife for indicating contributions, available online at https://elifesciences.org/inside-elife/f39cfcf5/enabling-the-contributor-roles-taxonomy-for-authorcontributions.

While the original CRediT standard recommended a binary contribution facility, whereby contributors either did or did not fulfil each of the contributor roles, there has been some discussion about whether contributor role models need to also capture some information about the extent of the contribution. A number of journals are now experimenting with this, providing the ability of authors to indicate whether they provided a "lead", "equal", or "supporting" role for each contribution identified.

\section{Adoption}

Proposals to reform an aspect of the incentive system in academia should expect resistance, in part because people who succeeded based on the current system typically have the most power. But shifting to contributorship need not be a big leap and indeed, despite little resource allocated to supporting CRediT, CRediT has already seen substantial uptake. It seems that many already see it as a practical and important move towards a more transparent, up-to-date, and useful conceptualisation of authorship [21]. This move can be done in small steps. To this end, here is a list of actions that a journal can take:

(1) Eliminate the requirement for a writing-related contribution for authorship.

(2) Require researchers to indicate the contribution of each author (not necessarily with CRediT).

(3) Provide the option for researchers to use CRediT.

(4) Require that researchers use CRediT.

(5) Amend guidelines to allow inclusion of those whose contribution is solely to securing funding, or other aspects of the CRediT taxonomy not noted in ICMJE guidelines.

Many journals have already implemented actions 1 or 2 above. The journals of the Nature Publishing Group [9], for example, have already adopted the McNutt et al. authorship criteria. The National Academies of Science maintain a list of journals that have adopted the recommended authorship criteria in whole or in part (www.nasonline.org/about-nas/Transparency_ Author_Contributions.html). Hesitant journals may be reassured by the length of that list.

The Nature Publishing Group journals also require authors to describe their contributions. Adoption of the CRediT taxonomy would standardize these descriptions to be comparable to those of other publishers and make them more machine readable, via JATS metadata. 
The popular PLoS stable of open access journals already require that all authors use the CRediT taxonomy, making it clear who did what [22]. However, they continue to use the writing-mandatory ICMJE criteria, meaning that many people who made substantial contributions may not be listed as authors. The PLoS guidelines do additionally state that "Contributors who do not meet the criteria for authorship should be mentioned in the Acknowledgments", however, they do not use the CRediT taxonomy in the Acknowledgments; instead it is written as free prose, and thus is not machine-readable.

Over twenty publishers are, at the time of this writing, listed at the CRediT site as having adopted CRediT. This does not mean that they all require authors to use the CRediT taxonomy in their journals. But they do have it as an option in their journal management system, and for most, at some of their journals researchers can at least opt to use it. Education of researchers and scientific societies about the option and its rationale may result in movement of more journals to join the PLoS journals, eLife, and F1000 platforms in requiring it.

Some journals have adopted the CRediT taxonomy, at least as an option for authors, without revising their writing-mandatory authorship guidelines. At the time of this writing, in addition to the PLoS journals, this group includes the Journal of Cell Biology, the Journal of Experimental Medicine, and the Journal of General Physiology. Because of their continued use of writing-mandatory guidelines, at least one of the two writing-related boxes must be ticked for every author. This is a way that journals can move toward contributorship where there is not agreement that the mandate on writing-related contributions should be removed. This step does provide some benefits-in the words of the journal eLife, "As more journals adopt it and the contributions are showcased by ORCID and others, we hope to see funding and promotion decisions explicitly reward the specific and varied contributions of researchers, rather than the number of first-author publications." With authorship order a weak and ambiguous indicator of researchers' contributions, the CRediT system should vastly improve things.

Normally, a barrier to the adoption of authorship reform is that experienced researchers are unlikely to read authorship criteria. An advantage of the CRediT taxonomy is that it is typically implemented with a hierarchy of checkboxes (Figure 1). As a result, even those who do not read the policy may end up conforming to a good portion of it. And the need to navigate the checkboxes is likely to induce some researchers to read the associated text. At the least, more would do so than the number who otherwise read authorship criteria.

Some scientific journals are more conservative than others and will be unlikely to change until it is clear that a large number of authors desire that change. Authors in an area may organize joint letters to editors or scientific societies to make the case for change, e.g., [23]. Individual authors can also send a message, for example by writing a list of the contributions, using the CRediT taxonomy, in the free-text acknowledgments section of their manuscripts ${ }^{1}$.

Authorship criteria today in medicine and many other fields are not simple, as they typically involve the combination of a mandatory writing requirement with other criteria, the nature of which (such as being optional or required) vary from journal to journal. The idea of indicating who did what is simpler. There is still the difficult issue of how substantive a contribution needs to be for a researcher to be credited, but this is an issue that any system faces. There seems to be little reason to not move toward a contributorship model; it provides a more inclusive and realistic recognition of their research contributions.

Funding: This research received no external funding.

Acknowledgments: I thank Liz Allen, Ven Popov, and Simine Vazire for comments.

Conflicts of Interest: The author declares no conflict of interest.

1 I thank an anonymous reviewer for suggesting this. 


\section{References}

1. International Committee of Medical Journal Editors. Defining the Role of Authors and Contributors. Updated December 2018. Available online: http://www.icmje.org/icmje-recommendations.pdf (accessed on 20 June 2019).

2. American Psychological Association. Publication Practices \& Responsible Authorship. Available online: https://www.apa.org/research/responsible/publication/ (accessed on 11 June 2019).

3. Rennie, D.; Yank, V.; Emanuel, L. When authorship fails: A proposal to make contributors accountable. JAMA 1997, 278, 579-585. [CrossRef] [PubMed]

4. Patience, G.S.; Galli, F.; Patience, P.A.; Boffito, D.C. Intellectual contributions meriting authorship: Survey results from the top cited authors across all science categories. PLoS ONE 2019, 14, e0198117. [CrossRef] [PubMed]

5. Resnik, D.B. A proposal for a new system of credit allocation in science. Sci. Eng. Ethics 1997, 3, $237-243$. [CrossRef]

6. McNutt, M.K.; Bradford, M.; Drazen, J.M.; Hanson, B.; Howard, B.; Jamieson, K.H.; Kiermer, V.; Marcus, E.; Pope, B.K.; Schekman, R. Transparency in authors' contributions and responsibilities to promote integrity in scientific publication. Proc. Natl. Acad. Sci. USA 2018, 115, 2557-2560. [CrossRef] [PubMed]

7. Nature Publishing Group. Nature Research Journals' Authorship Policy. Available online: https://www. nature.com/authors/policies/authorship.html (accessed on 11 June 2019).

8. Resnik, D.B.; Tyler, A.M.; Black, J.R.; Kissling, G. Authorship policies of scientific journals. J. Med. Ethics 2016, 42, 199-202. [CrossRef]

9. Authorship policies. Nature 2009, 458, 1078. [CrossRef]

10. Greenland, P.; Fontanarosa, P.B. Ending Honorary Authorship. Science 2012, 337, 1019. [CrossRef]

11. Kumar, S. Ethical Concerns in the Rise of Co-Authorship and Its Role as a Proxy of Research Collaborations. Publications 2018, 6, 37. [CrossRef]

12. Cutas, D.; Shaw, D. Writers blocked: On the wrongs of research co-authorship and some possible strategies for improvement. Sci. Eng. Ethics 2015, 21, 1315-1329. [CrossRef] [PubMed]

13. Shen, S.X. Negotiating authorship in Chinese universities: How organizations shape cycles of credit in science. Sci. Technol. Hum. Values 2016, 41, 660-685. [CrossRef]

14. Beaumont, M.A.; Rannala, B. The Bayesian revolution in genetics. Nat. Rev. Genet. 2004, 5, 251. [CrossRef] [PubMed]

15. Rylance, R. Grant giving: Global funders to focus on interdisciplinarity. Nature 2015, 525, 313-315. [CrossRef] [PubMed]

16. Lyall, C.; Bruce, A.; Marsden, W.; Meagher, L. The role of funding agencies in creating interdisciplinary knowledge. Sci. Public Policy 2013, 40, 62-71. [CrossRef]

17. ADAlthousePhD. [Twitter Post]. It's Kind of Up to Individual Institutions to Police Their Faculty, but Complaining about This Can Make You a Pariah or Get You a Reputation as Being "Difficult" (for the Outrageous Demand of Wanting Credit for Your Work ... ). Available online: https://twitter. com/ADAlthousePhD/status/1104003152964132865 (accessed on 8 March 2019).

18. Ridley, A. Now Is the Time for a Team-Based Approach to Team Science. The Academy of Medical Sciences Website. 2019. Available online: https://acmedsci.ac.uk/more/news/now-is-the-time-for-a-team-basedapproach-to-team-science (accessed on 11 May 2019).

19. Allen, L.; Scott, J.; Brand, A.; Hlava, M.; Altman, M. Publishing: Credit where credit is due. Nature 2014, 508, 312-313. [CrossRef]

20. Brand, A.; Allen, L.; Altman, M.; Hlava, M.; Scott, J. Beyond authorship: Attribution, contribution, collaboration, and credit. Learn. Publ. 2015, 28, 151-155. [CrossRef]

21. Allen, L.; O'Connell, A.; Kiermer, V. How can we ensure visibility and diversity in research contributions? How the Contributor Role Taxonomy (CRediT) is helping the shift from authorship to contributorship. Learn. Publ. 2019, 32, 71-74. [CrossRef] 
22. PLoS. Available online: https://journals.plos.org/plosone/s/authorship (accessed on 13 March 2019).

23. Holcombe, A.O.; Vazire, S.; Chartier, C.R. Replace Journals' Writing-Based Authorship Guidelines with a Contributorship Model. In Proceedings of the the Annual Meeting of the Society for the Improvement of Psychological Science, Rotterdam, The Netherlands, 7-9 July 2019. 